\title{
Database of Multichannel In-Ear and Behind-the-Ear Head-Related and Binaural Room Impulse Responses
}

\author{
H. Kayser, S. D. Ewert, J. Anemüller, T. Rohdenburg, V. Hohmann, and B. Kollmeier
}

Medizinische Physik, Universität Oldenburg, 26111 Oldenburg, Germany

Correspondence should be addressed to H. Kayser, hendrik.kayser@uni-oldenburg.de

Received 15 December 2008; Accepted 4 June 2009

Recommended by Hugo Fastl

\begin{abstract}
An eight-channel database of head-related impulse responses (HRIRs) and binaural room impulse responses (BRIRs) is introduced. The impulse responses (IRs) were measured with three-channel behind-the-ear (BTEs) hearing aids and an in-ear microphone at both ears of a human head and torso simulator. The database aims at providing a tool for the evaluation of multichannel hearing aid algorithms in hearing aid research. In addition to the HRIRs derived from measurements in an anechoic chamber, sets of BRIRs for multiple, realistic head and sound-source positions in four natural environments reflecting dailylife communication situations with different reverberation times are provided. For comparison, analytically derived IRs for a rigid acoustic sphere were computed at the multichannel microphone positions of the BTEs and differences to real HRIRs were examined. The scenes' natural acoustic background was also recorded in each of the real-world environments for all eight channels. Overall, the present database allows for a realistic construction of simulated sound fields for hearing instrument research and, consequently, for a realistic evaluation of hearing instrument algorithms.
\end{abstract}

Copyright (C) 2009 H. Kayser et al. This is an open access article distributed under the Creative Commons Attribution License, which permits unrestricted use, distribution, and reproduction in any medium, provided the original work is properly cited.

\section{Introduction}

Performance evaluation is an important part of hearing instrument algorithm research since only a careful evaluation of accomplished effects can identify truly promising and successful signal enhancement methods. The gold standard for evaluation will always be the unconstrained real-world environment, which comes however at a relatively high cost in terms of time and effort for performance comparisons.

Simulation approaches to the evaluation task are the first steps in identifying good signal processing algorithms. It is therefore important to utilize simulated input signals that represent real-world signals as faithfully as possible, especially if multimicrophone arrays and binaural hearing instrument algorithms are considered that expect input from both sides of a listener's head. The simplest approach to model the input signals to a multichannel or binaural hearing instrument is the free-field model. More elaborate models are based on analytical formulations of the effect that a rigid sphere has on the acoustic field $[1,2]$.

Finally, the synthetic generation of multichannel input signals by means of convolving recorded (single-channel) sound signals with impulse responses (IRs) corresponding to the respective spatial sound source positions, and also depending on the spatial microphone locations, represents a good approximation to the expected recordings from a realworld sound field. It comes at a fraction of the cost and with virtually unlimited flexibility in arranging different acoustic objects at various locations in virtual acoustic space if the appropriate room-, head-, and microphone-related impulse responses are available.

In addition, when recordings from multichannel hearing aids and in-ear microphones in a real acoustic background sound field are available, even more realistic situations can be produced by superimposing convolved contributions from localized sound sources with the approximately omnidirectional real sound field recording at a predefined mixing ratio. By this means, the level of disturbing background noise can be controlled independently from the localized sound sources.

Under the assumption of a linear and time-invariant propagation of sound from a fixed source to a receiver, the impulse response completely describes the system. All transmission characteristics of the environment and objects 
in the surrounding area are included. The transmission of sound from a source to human ears is also described in this way. Under anechoic conditions the impulse response contains only the influence of the human head (and torso) and therefore is referred to as head-related impulse response (HRIR). Its Fourier transform is correspondingly referred to as head-related transfer function (HRTF). Binaural headrelated IRs recorded in rooms are typically referred to as binaural room impulse responses (BRIRs).

There are several existing free available databases containing HRIRs or HRTFs measured on individual subjects and different artificial head-and-torso simulators (HATS) [3-6]. However these databases are not suitable to simulate sound impinging on hearing aids located behind the ears (BTEs) as they are limited to two-channel information recorded near the entrance of the ear canal. Additionally the databases do not reflect the influence of the room acoustics.

For the evaluation of modern hearing aids, which typically process 2 or 3 microphone signals per ear, multichannel input data are required corresponding to the real microphone locations (in the case of BTE devices behind the ear and outside the pinna) and characterizing the respective room acoustics.

The database presented here therefore improves over existing publicly available data in two respects: In contrast to other HRIR and BRIR databases, it provides a dummy-head recording as well as an appropriate number of microphone channel locations at realistic spatial positions behind the ear. In addition, several room acoustical conditions are included.

Especially for the application in hearing aids, a broad set of test situations is important for developing and testing of algorithms performing audio processing. The availability of multichannel measurements of HRIRs and BRIRs captured by hearing aids enables the use of signal processing techniques which benefit from multichannel input, for example, blind source separation, sound source localization and beamforming. Real-world problems, such as head shading and microphone mismatch [7] can be considered by this means.

A comparison between the HRTFs derived from the recorded HRIRs at the in-ear and behind-the-ear positions and respective modeled HRTFs based on a rigid spherical head is presented to analyze deviations between simulations and a real measurements. Particularly at high frequencies, deviations are expected related to the geometric differences between the real head including the pinnae and the model's spherical head.

The new database of head-, room- and microphonerelated impulse responses, for convenience consistently referred to as HRIRs in the following, contains six-channel hearing aid measurements (three per side) and additionally the in-ear HRIRs measured on a Brüel \& Kjær HATS [8] in different environments.

After a short overview of the measurement method and setup, the acoustic situations contained in the database are summarized, followed by a description of the analytical head model and the methods used to analyze the data. Finally, the results obtained under anechoic conditions are compared to synthetically generated HRTFs based on the

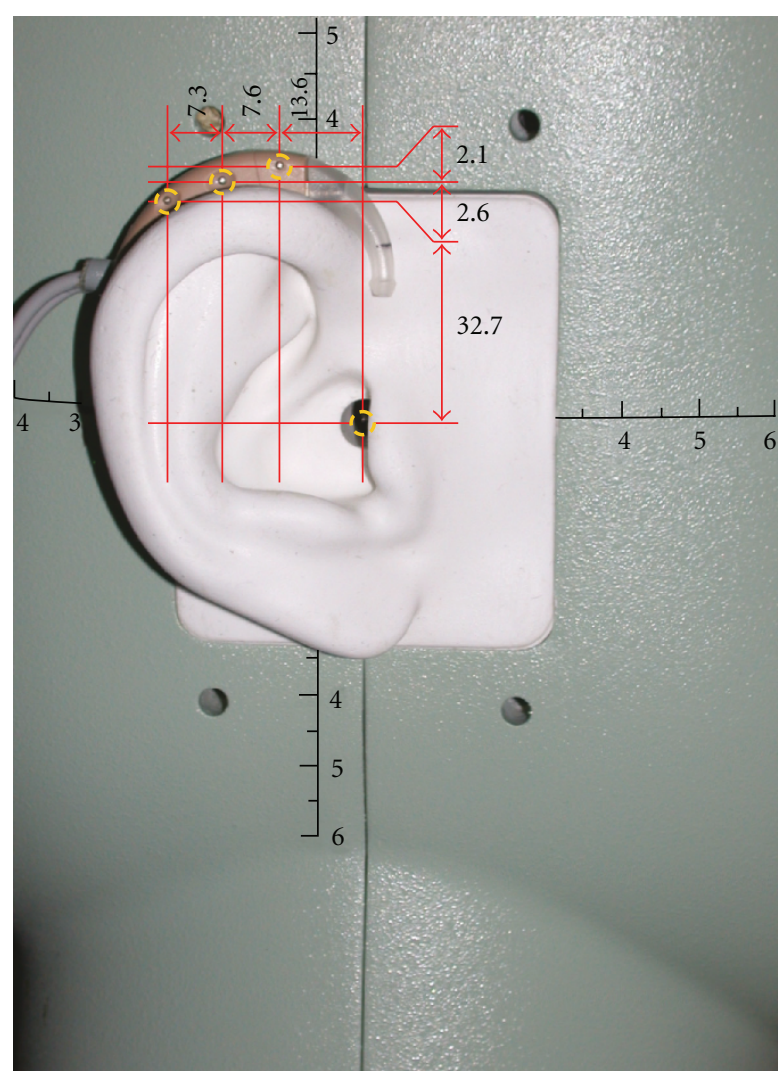

FIGURE 1: Right ear of the artificial head with a hearing aid dummy. The distances between the microphones of the hearing aids and the entrance to the earcanal on the artificial head are given in $\mathrm{mm}$.

model of a rigid sphere. The database is available under http://medi.uni-oldenburg.de/hrir/.

\section{Methods}

2.1. Acoustic Setup. Data was recorded using the head-andtorso simulator Brüel \& Kjær Type 4128C onto which the BTE hearing aids were mounted (see Figure 1). The use of a HATS has the advantage of a fixed geometry and thereby provides highly reproducible acoustic parameters. In addition to the microphones in the BTEs mounted on the HATS, it also provides internal microphones to record sound pressure near the location corresponding to the place of the human ear drum.

The head-and-torso simulator was used with artificial ears Brüel \& Kjær Type 4158C (right) and Type 4159C (left) including preamplifiers Type 2669. Recordings were carried out with the in-ear microphones and two threechannel BTE hearing aid dummies of type Acuris provided by Siemens Audiologische Technik GmbH, one behind each artificial ear, resulting in a total of 8 recording channels. The term "hearing aid dummy" refers to the microphone array of a hearing aid, housed in its original casing but without any of the integrated amplifiers, speakers or signal processors commonly used in hearing aids. 
The recorded analog signals were preamplified using a G.R.A.S. Power Module Type 12AA, with the amplification set to $+20 \mathrm{~dB}$ (in-ear microphones) and a Siemens custom-made pre-amplifier, with an amplification of $+26 \mathrm{~dB}$ on the hearing aid microphones. Signals were converted using a 24-bit multichannel AD/DAconverter (RME Hammerfall DSP Multiface) connected to a laptop (DELL Latitude 610D, Pentium M processor $@ 1.73 G h z, 1 G B R A M)$ via a PCMCIA-card and the digital data was stored either on the internal or an external hard disk. The software used for the recordings was MATLAB (MathWorks, Versions 7.1/7.2, R14/R2006a) with a professional tool for multichannel I/O and real-time processing of audio signals (SoundMex2 [9]).

The measurement stimuli for measuring a HRIR were generated digitally on the computer using MATLABscripts (developed in-house) and presented via the $\mathrm{AD} / \mathrm{DA}$ converter to a loudspeaker. The measurement stimuli were emitted by an active 2-channel coaxial broadband loudspeaker (Tannoy $800 \mathrm{~A} \mathrm{LH}$ ). All data was recorded at a sampling rate of $48 \mathrm{kHz}$ and stored at a resolution of $32 \mathrm{Bit}$.

2.2. HRIR Measurement. The HRIR measurements were carried out for a variety of natural recording situations. Some of the scenarios were suffering from relatively high levels of ambient noise during the recording. Additionally, at some recording sites, special care had to be taken of the public (e.g., cafeteria). The measurement procedure was therefore required to be of low annoyance while the measurement stimuli had to be played back at a sufficient level and duration to satisfy the demand of a high signalto-noise ratio imposed by the use of the recorded HRIRs for development and high-quality auralization purposes. To meet all requirements, the recently developed modified inverse-repeated sequence (MIRS) method [10] was used. The method is based on maximum length sequences (MLS) which are highly robust against transient noise since their energy is distributed uniformly in the form of noise over the whole impulse response [11]. Furthermore, the broadband noise characteristics of MLS stimuli made them suitable for presentation in the public rather than, for example, sine-sweep stimuli-based methods [12]. However, MLSs are known to be relatively sensitive to (even weak) nonlinearities in the measurement setup. Since the recordings at public sites required partially high levels reproduced by small scale and portable equipment, the risk of non-linear distortions was present. Inverse repeated sequences (IRS) are a modification to MLSs which show high robustness against even-order nonlinear distortions [13]. An IRS consists of two concatenated MLS $s(n)$ and its inverse:

$$
\operatorname{IRS}(n)=\left\{\begin{array}{ll}
s(n), & n \text { even, } \\
-s(n), & n \text { odd, }
\end{array} \quad 0 \leq n \leq 2 L,\right.
$$

where $L$ is the period of the generating MLS. The IRS therefore has a period of $2 L$. In the MIRS method employed here, IRSs of different orders are used in one measurement process and the resulting impulse responses of different lengths are median-filtered to further suppress the effect of uneven-order nonlinear distortions after the following scheme: A MIRS consists of several successive IRS of different orders. In the evaluation step, the resulting periodic IRs of the same order were averaged yielding a set of IRs of different orders. The median of these IRs was calculated and the final IR was shortened to length corresponding to the lowest order. The highest IRS order in the measurements was 19, which is equal to a length of 10.92 seconds at the used sampling rate of $48 \mathrm{kHz}$. The overall MIRS was 32.77 seconds in duration and the calculated raws IRs were 2.73 seconds corresponding to 131072 samples.

The MIRS method combines the advantages of MLS measurements with high immunity against non-linear distortions. A comparison of the measurement results to an efficient method proposed by Farina [12] showed that the MIRS technique achieves competitive results in anechoic conditions with regard to signal-to-noise ratio and was better suited in public conditions (for details see [10]).

The transfer characteristics of the measurement system was not compensated for in the HRIRs presented here, since it does not effect the interaural and microphone array differences. The impulse response of the loudspeaker measured by a probe microphone at the HATS position in the anechoic chamber is provided as part of the database.

2.3. Content of the Database. A summary of HRIR measurements and recordings of ambient acoustic backgrounds (noise) is found in Table 1.

2.3.1. Anechoic Chamber. To simulate a nonreverberant situation, the measurements were conducted in the anechoic chamber of the University of Oldenburg. The HATS was fixed on a computer-controlled turntable (Brüel \& Kjær Type 5960C with Controller Type 5997) and placed opposite to the speaker in the room as shown in Figure 2. Impulse responses were measured for distances of $0.8 \mathrm{~m}$ and $3 \mathrm{~m}$ between speaker and the HATS. The larger distance corresponds to a far-field situation (which is, e.g., commonly required by beam-forming algorithms) whereas for the smaller distance near-field effects may occur. For each distance, 4 angles of elevation were measured ranging from $-10^{\circ}$ to $20^{\circ}$ in steps of $10^{\circ}$. For each elevation the azimuth angle of the source to the HATS was varied from $0^{\circ}$ (front) to $-180^{\circ}$ (left turn) in steps of $5^{\circ}$ (cf. Figure 3$)$. Hence, a total of $296(=37 \times 4 \times 2)$ sets of impulse responses were measured.

2.3.2. Office I. In an office room at the University of Oldenburg similar measurements were conducted, covering the systematic variation of the sources' spatial positions. The HATS was placed on a desk and the speaker was moved in the front hemisphere (from $-90^{\circ}$ to $+90^{\circ}$ ) at a distance of $1 \mathrm{~m}$ with an elevation angle of $0^{\circ}$. The step size of alteration of the azimuth angle was $5^{\circ}$ as for the anechoic chamber.

For this environment only the BTE channels were measured.

A detailed sketch of the recording setup for this and the other environments is provided as a part of the database. 
TABLE 1: Summary of all measurements of head related impulse responses and recordings of ambient noise. In the Office I environment (marked by the asterisk) only the BTE channels were measured.

\begin{tabular}{lcc}
\hline Environment & HRIR sets measured & Sounds recorded \\
\hline Anechoic chamber & 296 & - \\
Office I & $37^{*}$ & 12 recordings of ambient noise, total duration 19 min \\
Office II & 8 & 2 recordings of ambient noise, total duration 14 min \\
Cafeteria & 12 & 1 recording of ambient noise, total duration 24 min \\
Courtyard & 12 & 57 min of different ambient noises \\
Total & 365 & \\
\hline
\end{tabular}

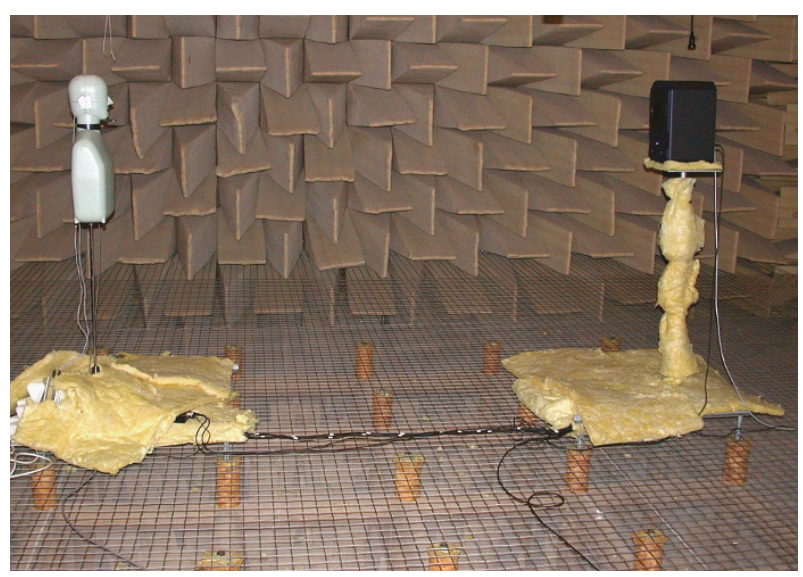

FIGURE 2: Setup for the impulse response measurement in the anechoic room. Additional damping material was used to cover the equipment in the room in order to avoid undesired reflections.

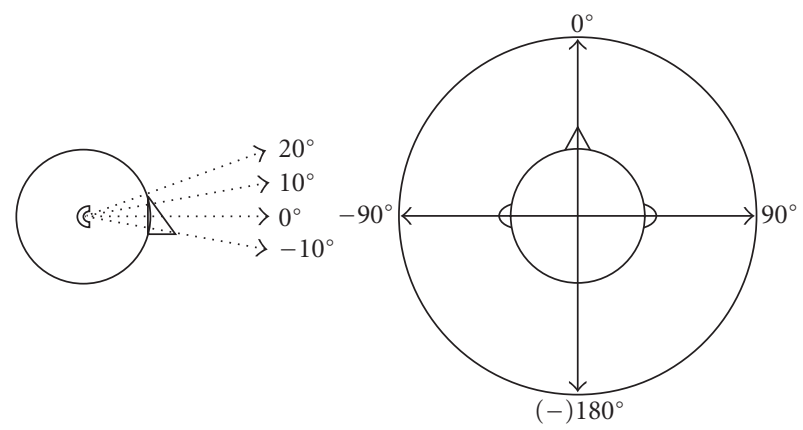

Figure 3: Coordinate systems for elevation angles (left-hand sketch) and azimuth angles (right-hand sketch).

2.3.3. Office II. Further measurements and recordings were carried out in a different office room of similar size. The head-and-torso simulator was positioned on a chair behind a desk with two head orientations of $0^{\circ}$ (looking straight ahead) and $90^{\circ}$ (looking over the shoulder). Impulse responses were measured for four different speaker positions (entrance to the room, two different desk conditions and one with a speaker standing at the window) to allow for simulation of sound sources at typical communication positions. For measurements with the speaker positioned at the entrance the door was opened and for the measurement at the window this was also open. For the remaining measurements door and window were closed to reduce disturbing background noise from the corridor and from outdoors. In total, this results in 8 sets of impulse responses.

Separate recordings of real office ambient sound sources were performed: a telephone ringing (30 seconds recorded for each head orientation) and keyboard typing at the other office desks (3 minutes recorded for each head orientation). The noise emitted by the ventilation, which is installed in the ceiling, was recorded for 5 minutes (both head orientations). Additionally, the sound of opening and closing the door was recorded 15 times.

2.3.4. Cafeteria. 12 sets of impulse responses were measured in the fully occupied cafeteria of the natural sciences campus of the University of Oldenburg. The HATS was used to measure the impulse responses from different positions and to collect ambient sound signals from the cafeteria. The busy lunch time hour was chosen to obtain realistic conditions.

The ambient sounds consisted mainly of unintelligible babble of voices from simultaneous conversations all over the place, occasional parts of intelligible speech from nearby speakers and the clanking of dishes and chairs scratching on the stone floor.

2.3.5. Courtyard. Measurements in the courtyard of the natural sciences campus of the University of Oldenburg were conducted analogous to the Office II and Cafeteria recordings described above. A path for pedestrians and bicycles crosses this yard. The ambient sounds consist of snippets of conversation between people passing by, foot steps and mechanical sounds from bicycles including sudden events such as ringing and squeaking of brakes. Continuous noise from trees and birds in the surrounding was also present.

2.4. Analytical Model and Data Analysis Methods. The characteristics of HRIRs and the corresponding HRTFs originates from diffraction, shading and resonances on the head and on the pinnae [14]. Also reflections and diffractions of the sound from the torso influence the HRTFs.

An analytical approximative model of the sound propagation around the head is the scattering of sound by a rigid sphere whose diameter $a$ equals the diameter of a human head. This is a simplification as the shoulders and the 
pinnae are neglected and the head is regarded as spherically symmetric.

The solution in the frequency domain for the diffraction of sound waves on a sphere traces back to Lord Rayleigh [15] in 1904. He derived the transfer function $H(\infty, \theta, \mu)$ dependent on the normalized frequency $\mu=k a=2 \pi \mathrm{fa} / \mathrm{c}$ ( $c$ : sound velocity) for an infinitely distant source impinging at the angle $\theta$ between the surface normal at the observation point and the source:

$$
H(\infty, \theta, \mu)=\frac{1}{\mu^{2}} \sum_{m=0}^{\infty} \frac{(-i)^{m-1}(2 m+1) P_{m}(\cos \theta)}{h_{m}^{\prime}(\mu)}
$$

where $P_{m}$ denotes the Legendre polynomials, $h_{m}$ the $m$ thorder spherical Hankel function and $h_{m}^{\prime}$ its derivative. Rabinowitz et al. [16] presented a solution for a point source in the distance $r$ from the center of the sphere:

$$
H(r, \theta, \mu)=-\frac{r}{a \mu} e^{-i \mu r / a} \Psi
$$

with

$$
\Psi=\sum_{m=0}^{\infty} \frac{(2 m+1) P_{m}(\cos \theta) h_{m}(\mu r / a)}{h_{m}^{\prime}(\mu)}, \quad r>\alpha .
$$

2.4.1. Calculation of Binaural Cues. The binaural cues, namely the interaural level difference (ILD), the interaural phase difference (IPD) and derived therefrom the interaural time difference (ITD), can be calculated in the frequency domain from a measured or simulated HRTF [17]. If $H_{l}(\alpha, \varphi, f)$ denotes the HRTF from the source to the left ear and $H_{r}(\alpha, \varphi, f)$ the transmission to the right ear, the interaural transfer function (ITF) is given by

$$
\operatorname{ITF}(\alpha, \varphi, f)=\frac{H_{l}(\alpha, \varphi, f)}{H_{r}(\alpha, \varphi, f)},
$$

with $\alpha$ and $\varphi$ the azimuth and elevations angles, respectively, as shown in Figure 3 and $f$ representing the frequency in $\mathrm{Hz}$.

The ILD is determined by

$$
\operatorname{ILD}(\alpha, \varphi, f)=20 \cdot \log _{10}(|\operatorname{ITF}(\alpha, \varphi, f)|) .
$$

The IPD can also be calculated from the ITF. Derivation with respect to the frequency $f$ yields the ITD which equals the group delay between both ears:

$$
\begin{aligned}
\operatorname{IPD}(\alpha, \varphi, f) & =\arg (\operatorname{ITF}(\alpha, \varphi, f)), \\
\operatorname{ITD}(\alpha, \varphi, f) & =-\frac{1}{2 \pi} \frac{d}{d f} \operatorname{IPD}(\alpha, \varphi, f)
\end{aligned}
$$

Kuhn presented the limiting cases for (2) in [2]. For low frequencies corresponding to the case $k a \ll 1$ the transfer function of the spherical head model simplifies to

$$
H_{l f}(\infty, \theta, \mu) \approx 1-i \frac{3}{2} \mu \cos \theta
$$

This yields an angle of incidence independent ILD of $0 \mathrm{~dB}$ and an angle dependent IPD. In the coordinate system given in Figure 3 the IPD amounts to

$$
\operatorname{IPD}_{l f}(\alpha) \approx 3 k a \sin \alpha,
$$

which results in

$$
\operatorname{ITD}_{l f}(\alpha) \approx \frac{6 \pi a}{c} \sin \alpha
$$

For high frequencies the propagation of the waves is described as "creeping waves" traveling around the sphere with approximately the speed of sound. In this case, the ITD can be derived from geometric treatment by the difference between the distance from the source to the left ear and the right ear considering the path along the surface of the sphere [18]:

$$
\operatorname{ITD}_{h f} \approx \frac{2 \pi a}{c}(\sin (\alpha)+\alpha)
$$

With the approximation $\alpha \approx \sin \alpha$, (tolerating an error of $5.5 \%$ for $\alpha=135^{\circ}$ and an error of $11 \%$ for $\alpha=150^{\circ}$ [2]) (11) yields:

$$
\operatorname{ITD}_{h f}(\alpha) \approx \frac{4 \pi a}{c} \sin \alpha,
$$

which equals $2 / 3$ times the result of (10).

In practice, the measured IPD is contaminated by noise. Hence, the data was preprocessed before the ITD was determined. First, the amplitude of the ITF was equalized to unity by calculating the sign of the complex valued ITF:

$$
\begin{aligned}
\widehat{\operatorname{ITF}}(\alpha, \varphi, f) & =\operatorname{sign}(\operatorname{ITF}(\alpha, \varphi, f)) \\
& =\frac{\operatorname{ITF}(\alpha, \varphi, f)}{|\operatorname{ITF}(\alpha, \varphi, f)|}
\end{aligned}
$$

The result was then smoothed applying a sliding average with a 20-samples window. The ITD was obtained for a specific frequency by calculating the weighted mean of the ITD (derived from the smoothed IPD) for a chosen range around this frequency. As weighting function the coherence function $\gamma$ was used, respectively a measure for the coherence $\gamma^{n}$ which is obtained from

$$
\gamma^{n}=\underbrace{|\widehat{\operatorname{ITF}}(\alpha, \varphi, f)|^{n}}_{\text {smoothed }} .
$$

The function was raised to the power of $n$ to control the strength of suppression of data with a weak coherence. In the analysis $n=6$ turned out to be a suitable choice.

\section{Results}

3.1. Quality of the Measurements. As evaluation of the quality, the signal-to-noise ratio (SNR) of the measured impulse responses was calculated for each environment. The average noise power was estimated from the noise floor 
$i r_{\text {noise }}(t)$ for the interval $T_{\text {end }}$ at end of the measured IR, where the IR has declined below the noise level. The duration of the measured IRs was sufficient to assume that only noise was present in this part of the measured IR. With the average power estimated for the entire duration $T=2.73 \mathrm{~s}$ of the measured IR, $\operatorname{ir}(t)$, the SNR was calculated as

$$
\mathrm{SNR}=10 \log _{10} \frac{\left\langle i r^{2}(t)\right\rangle_{T}}{\left\langle i r_{\text {noise }}^{2}(t)\right\rangle_{T_{\text {end }}}},
$$

where $\langle\cdot\rangle$ denotes the temporal average.

The results are given in Table 2.

3.2. Reverberation Time of the Different Environments. The reverberation time $T_{60}$ denotes the time that it takes for the signal energy to decay by $60 \mathrm{~dB}$ after the playback of the signal is stopped. It was estimated from a room impulse response of duration $T$ employing the method of Schroeder integration [19]. In the Schroeder integration, the energy decay curve (EDC) is obtained by reverse-time integration of the squared impulse response:

$$
\operatorname{EDC}(t)=10 \log _{10} \frac{\int_{t}^{T} i r^{2}(\tau) d \tau}{\int_{0}^{T} i r^{2}(\tau) d \tau} .
$$

The noise contained in the measured IR is assumed to spread equally over the whole measured IR and thus leads to a linearly decreasing offset in the EDC. A correction for the noise is introduced by fitting a linear curve to the pure noise energy part at the end of the EDC, where the IR has vanished. Subsequently the linear curve, representing the effect of noise, is subtracted from the EDC yielding the pure IR component.

Generally, an exponential decay in time is expected and the decay rate was found by fitting an exponential curve to the computed decay of energy [20]. An example EDC is shown in Figure 4. The first steeply sloped part of the curve results from the decay of the energy of direct sound (early decay) fading at about 0.1 seconds to the part resulting from the diffuse reverberation tail of the IR. An exponential curve is fitted (linear in semilogarithmic presentation) to the part of the EDC corresponding to the reverberation tail. The $T_{60}$ time is then determined from the fitted decay curve. The estimated $T_{60}$ times of the different environments are given in Table 3.

\subsection{Comparison to the Analytical Model of A Rigid Sphere.} Duda and Martens provide pseudocode for the evaluation of (3) for the calculation of angle- and range-dependent transfer functions of a sphere in [1]. The behavior of the theoretical solution was also explored in detail within their work and compared to measurements carried out on a bowling ball. The pseudocode was implemented in MATLAB and 8-channel HRTFs were calculated for the microphone positions corresponding to the entrances of the ear canals of the HATS and the positions of the BTE hearing aid microphones on the artificial head.

In the following analysis, the measured HRTFs (obtained from the measured HRIRs) are compared to the data

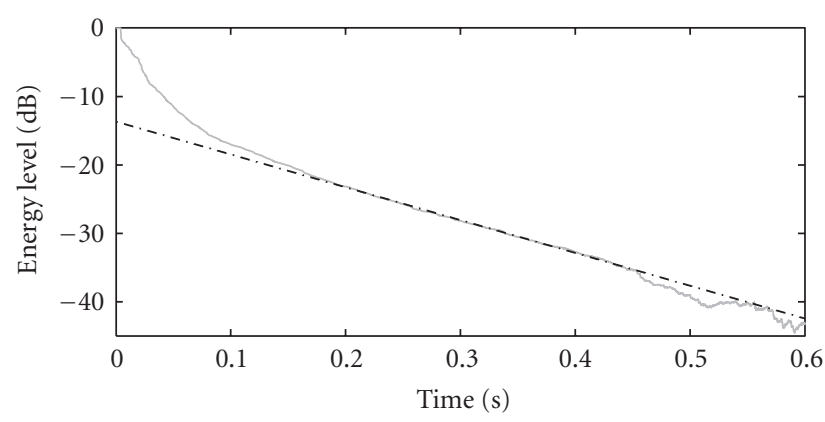

Figure 4: Energy decay curve calculated using the method of Schroeder integration from a impulse response of the cafeteria (solid) and linear fit (dashed) to estimate the reverberation time $T_{60}$.

TABLE 2: Mean SNR values of the impulse response measurements in the different environments.

\begin{tabular}{lc}
\hline Environment & SNR $(\mathrm{dB})$ \\
\hline Anechoic chamber & 104.8 \\
Office II & 94.7 \\
Cafeteria & 75.6 \\
Courtyard & 86.1 \\
\hline
\end{tabular}

TABLE 3: Reverberation time of the different environments.

\begin{tabular}{lc}
\hline Environment & $T_{60}(\mathrm{~ms})$ \\
\hline Anechoic chamber & $<50^{(1)}$ \\
Office II & 300 \\
Cafeteria & 1250 \\
Courtyard & 900 \\
\hline
\end{tabular}

(1) The reverberation time estimate is limited by decay of the impulse response of the vented loudspeaker system with a cut-off frequency of about $50 \mathrm{~Hz}$.

modeled for a rigid sphere and also differences between the in-ear HRTFs and the BTE hearing aids HRTFs are considered. It is analyzed to which extend a spherical head model is suitable to describe the sound incidence to the BTE hearing aids regarding binaural cues and properties in the time domain. The HRTFs from the anechoic room for the distance of $3 \mathrm{~m}$ and an elevation angle of $0^{\circ}$ are compared to the predictions of the model for a rigid sphere. The measured results displayed in the figures were smoothed to obtain a more articulate presentation. For this purpose, the HRTFs were filtered using a sliding rectangular window with a 1/12octave width.

Figure 5 shows exemplary transfer functions obtained for an azimuth angle of $-45^{\circ}$. On the left side, the measured HRTFs are shown, on the right side the theoretical curves for a spherical head without torso. These were calculated for the microphone positions corresponding to the measurement setup as shown in Figure 1, whereby only the azimuth angles were taken into account and the slight differences in elevation were neglected. In the low-frequency range up to $1 \mathrm{kHz}$, the dotted curves on the left and the right side have a similar course except for a stronger ripple of the measured 


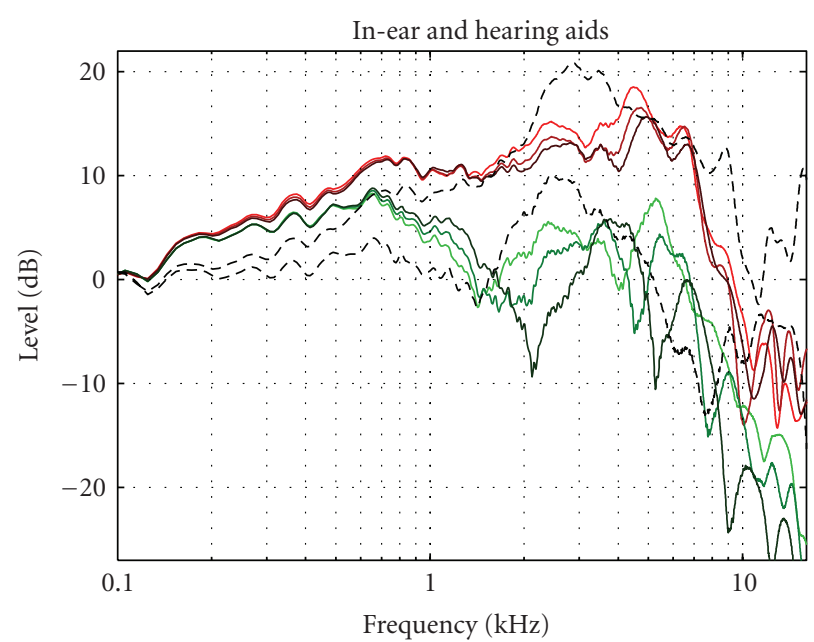

(a)

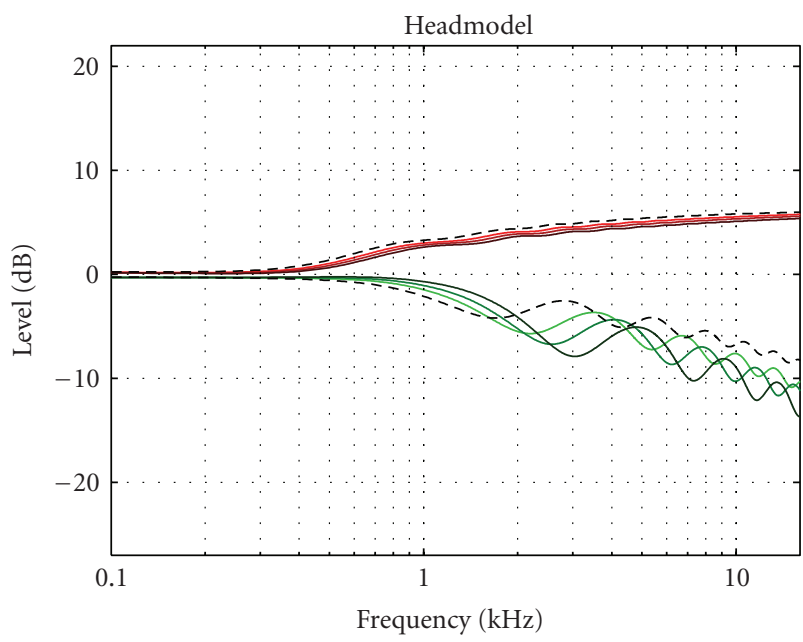

(b)

FIgURE 5: Measured HRTFs (a) (log-magnitude) from the in-ear (dashed) and the hearing aid microphones (solid) and corresponding logmagnitude transfer functions calculated by the model for an ideal rigid sphere (b). The angle of incidence is $-45^{\circ}$. The set of the upper four curves display the HRTFs from the left side of the artificial head, the lower set is obtained from the right side. The light colored lines represent the front hearing aid microphones and the dark lines the rearmost ones. A level of $0 \mathrm{~dB}$ corresponds to the absence of head-effects.

data. Level differences due to the transmission characteristics of the small hearing aid microphones (solid lines) which strongly deviates from a flat course are observed.

In the middle frequency range, both sides are still correlated, but the characteristic notches and maxima are much more prominent in the measurements. The intersection points of the separate curves remain similar, but the variation of the level and the level differences between the microphones are much stronger. The results of the in-ear measurements show a raise of $10 \mathrm{~dB}$ to $15 \mathrm{~dB}$ in comparison to the theoretical levels, due to resonances in the ear canal.

Above $7 \mathrm{kHz}$, effects like shadowing and resonance from the structure of the head which are not present in the head model have a strong influence.

In the following, the ITDs and ILDs obtained from the measurements are examined in more detail.

3.3.1. ILD. The ILDs from the inner ear microphones and one pair of the hearing aid microphones are shown in Figure 6 for a subset of azimuth angles (solid lines) along with the according curves obtained from the model (dashed lines).

As indicated in the previous figure, the measurements and the model show a similar behavior up to a frequency of about $3 \mathrm{kHz}$. Above this value, the influence of the head and the torso become obvious resulting in a strong ripple especially for the inner ear measurements which include also the effects of the pinnae and the ear canals.

Above a frequency of $9 \mathrm{kHz}$, alignment errors and microphone mismatch become obvious. This is indicated by the deviation of the ILD from the $0 \mathrm{~dB}$ line for sound incidence from $0^{\circ}$ and $-180^{\circ}$.

For the ILDs of the in-ear measurements it is obvious that the measured ILD is much bigger than the model ILD for sound incidence from the front left side $\left(-30^{\circ}\right.$ to $\left.-90^{\circ}\right)$ in the frequency range above $4 \mathrm{kHz}$. If the sound impinges from behind, notches are observable at $3 \mathrm{kHz}$ for $-120^{\circ}$ and at nearly $4 \mathrm{kHz}$ at $-150^{\circ}$ in the measured ILD when compared to the model ILD. This effect is not present in the ILDs between the hearing aids and therefore must originate from the pinnae.

3.3.2. ITD. The ITDs between the in-ear microphones and a microphone pair of the hearing aids were calculated as described in Section 2.4.1 within a range of $\pm 100 \mathrm{~Hz}$ to the center frequency. The results are shown in Figure 7, where the modeled data is also displayed.

For center frequencies of $125 \mathrm{~Hz}$ and $250 \mathrm{~Hz}$, the curves obtained from the measurements and the model are in good accordance. Above, for $0.5 \mathrm{kHz}$ and $1 \mathrm{kHz}$, deviations occur. Here, the ITDs calculated from the measurements are slightly higher than the theoretical values for the sphere. The determination of the azimuth angle is always ambiguous for a sound coming from the back or the front hemisphere. For the $2-\mathrm{kHz}$ curve, the ITD becomes also ambiguous for sound waves coming from the same hemisphere.

Another difference between the ILD for low and high frequencies is observable. For the lower frequencies, the time differences are larger than for higher frequencies at the same angle of incidence, corresponding to a larger effective head radius for low frequencies. This is in accordance with the findings of Kuhn [2] for an infinitely distant source described by (10) and (12).

3.3.3. Analysis in the Time Domain. Figure 8 shows HRIRs for a sound source impinging to the left side of the HATS. The angle of incidence ranges from $0^{\circ}$ to $360^{\circ}$ and, in this representation, is related to the angle of incidence to 


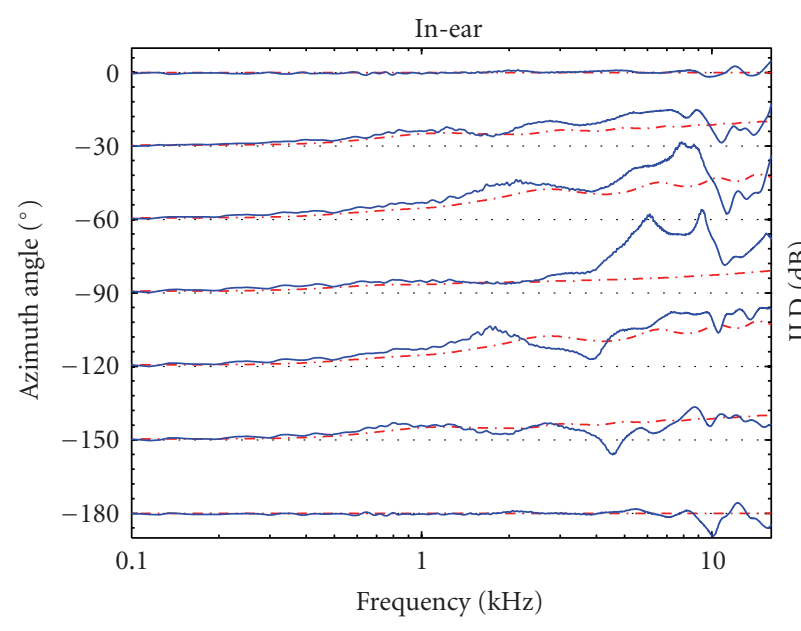

(a)

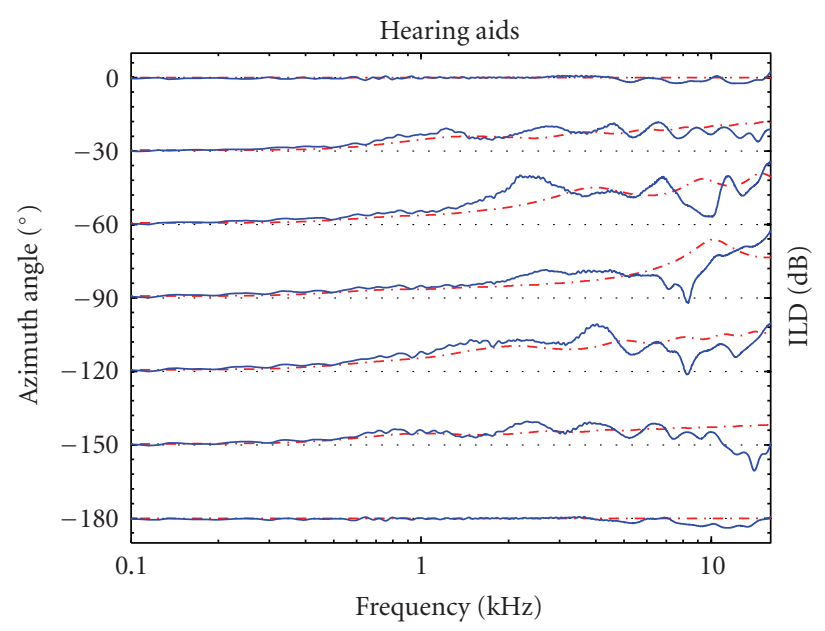

(b)

FIGURE 6: ILDs calculated from the measurements (solid lines) and the modeled HRTFs (dashed lines) for the in-ear microphones (a) and the front microphone pair of the hearing aids (b). One tick on the right ordinate corresponds to $6 \mathrm{~dB}$ level difference. The dashed straight lines mark the ILD of $0 \mathrm{~dB}$.

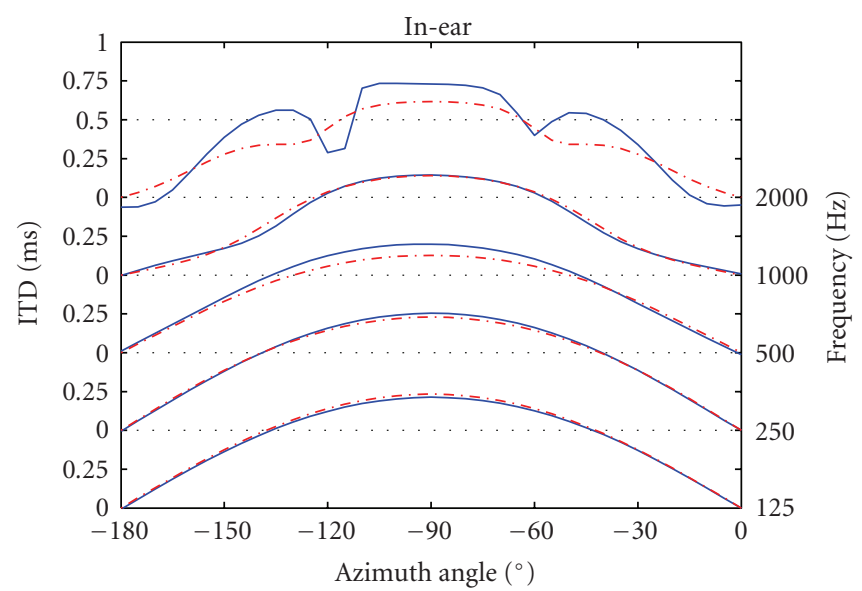

(a)

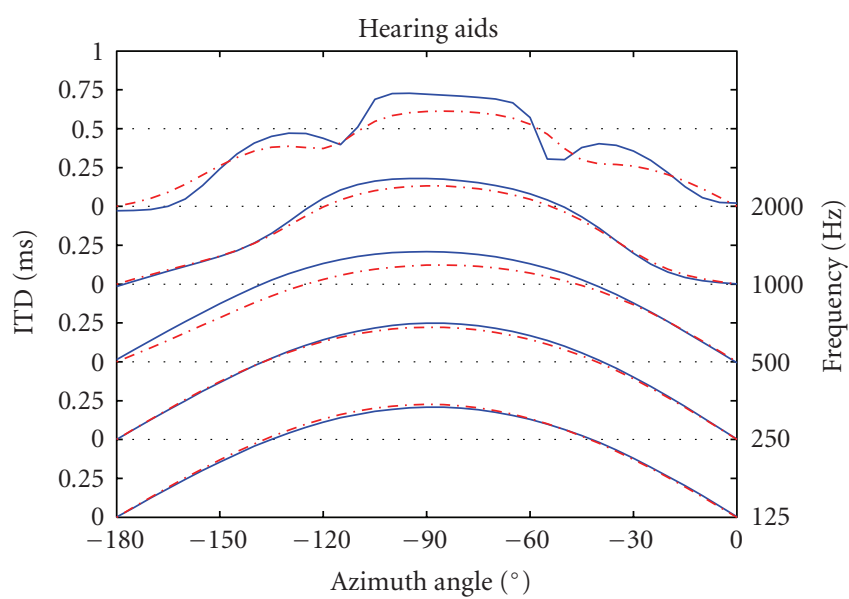

(b)

Figure 7: ITDs calculated from the measurements (solid lines) and the modeled HRTFs (dashed lines) for the in-ear microphones (a) and the front microphone pair of the hearing aids (b). The ITDs for the mid frequencies in octaves from $125 \mathrm{~Hz}$ to $2 \mathrm{kHz}$ are shown as indicated on the right-hand ordinate axis. An offset of 0.5 milliseconds is added to separate the curves from each other for a better overview. One tick on the left-hand ordinate is 0.25 milliseconds.

the microphones on the left side of the head for a better overview. This means, for an angle of $0^{\circ}$, the sound impinges perpendicularly to the hearing aid. The set of HRIRs is shown for the head model (a), the corresponding foremost hearing aid microphone on the left side (b) and the left inear microphone (c).

The data from the head model show a decreasing magnitude of the main peak with increasing angle of incidence up to $170^{\circ}$. For sound incidence from the opposite direction a peak is visible-the so-called "bright spot" which was also described by Duda and Martens [1].

The impulse responses of the hearing aid microphone also show a bright spot for sound incidence from $180^{\circ}$. The shape of the maximum peak formation is similar to the modeled data, but after the main peak additional delayed reflections occur. Early reflections are from the rim of the pinna as the delay lies within the range of travel time according to a distance of a few centimeters. A later dominant peak is attributed to strong reflections from the shoulders as it occurs 0.3 milliseconds to 0.5 milliseconds after the main peak which corresponds to a distance of about $13 \mathrm{~cm}$ to 20 $\mathrm{cm}$.

For the in-ear microphones these reflections are much more pronounced and have a finer structure. A bright spot is not apparent due to the asymmetry caused by the pinnae. 


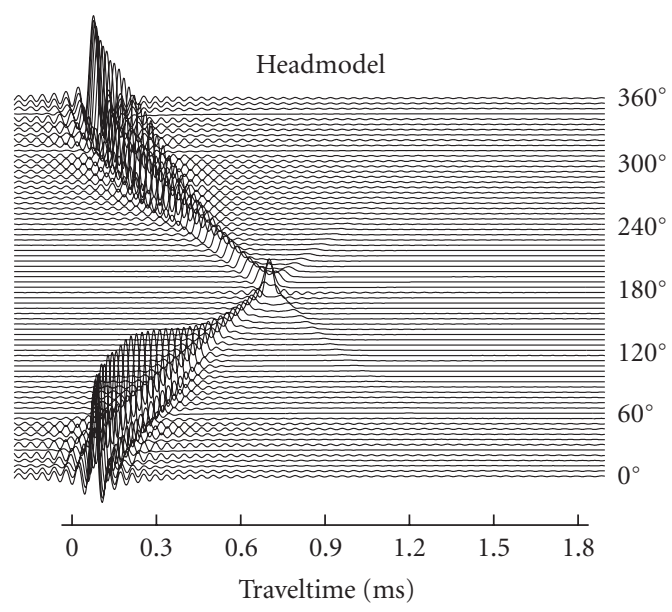

(a)

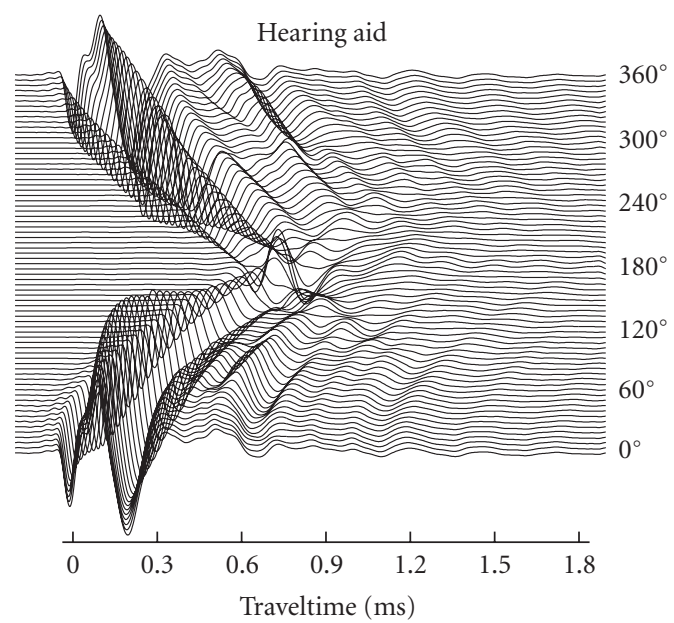

(b)

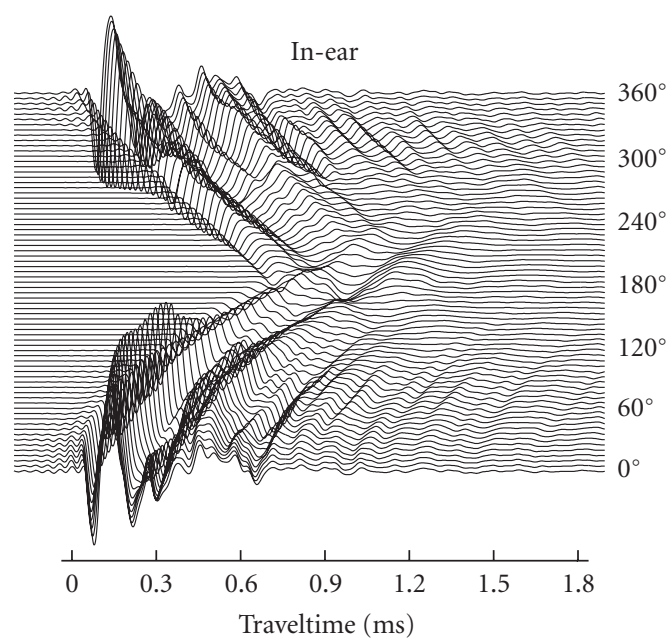

(c)

FIGURE 8: Head-related impulse responses for sound incidence to the left side of the artificial head. Data are shown for the head model (a), a hearing aid microphone (b) and the left in-ear microphone (c).

\section{Discussion and Conclusion}

A HRIR database was introduced, which is suited to simulate different acoustic environments for digital sound signal processing in hearing aids. A high SNR of the impulse responses was achieved even under challenging real-world recording conditions. In contrast to existing freely available databases, six-channel measurements of BTE hearing aids are included in addition to the in-ear HRIRs for a variety of source positions in a free-field condition and in different realistic reverberant environments. Recordings of the ambient sounds characteristic to the scenes are available separately. This allows for a highly authentic simulation of the underlying acoustic scenes.

The outcome of the analysis of the HRTFs from the anechoic room is in agreement with previous publications on HRTFs (e.g., [2]) and shows noticeable differences between the in-ear measurements and the data from the hearing aids. As expected, the ILDs derived from the spherical head model match the data from the hearing aids better than the data from the in-ear measurements. The modeled ILD fits the ILD between the hearing aids reasonably up to a frequency of $6 \mathrm{kHz}$. For the in-ear ILD, the limit is about $4 \mathrm{kHz}$.

In the frequency region above 4 to $6 \mathrm{kHz}$ significant deviations of the simulated data and the measurements occur. This shows, that modeling a head by a rigid sphere does not provide a suitable estimation of sound transmission to the microphone arrays in a BTE hearing aid and motivates the use of this database in hearing aid research, particularly for future hearing aids with extended frequency range.

It is expected that the data presented here will predominantly be used in the context of evaluation of signal processing algorithms with multi-microphone input such as beamformers or binaural algorithms. In such cases, very detailed knowledge about magnitude and phase behavior of the HRTFs might have to be provided as a-priori knowledge into signal processing algorithms. Even though the current HRTF data represent a "snapshot" of a single geometric head arrangement that would need to be adjusted to subjects on an individual basis, it can nevertheless be used as one specific realization to be accounted for in certain algorithms.

It is impossible to determine a-priori whether the detailed acoustic properties captured by realistic HRIRs/HRTFs are indeed significant for either evaluation or algorithm construction. However, the availability of the current database makes it possible to answer this question for each specific algorithm, acoustic situation and performance measure individually. Results from work based on our data [21] demonstrate that even for identical algorithms and spatial arrangements, different measures can show a significant performance increase (e.g., SNR enhancement) when realistic HRTFs are taken into account. Conversely, other measures (such as the speech reception threshold under binaural conditions) have been found to be largely invariant to the details captured by realistic models. In any case, the availability of the HRIR database presented here makes it possible to identify the range of realistic conditions 
under which an arbitrary hearing instrument algorithm performs well.

This "test-bed" environment also permits detailed comparison between different algorithms and may lead to a realistic de facto standard benchmark dataset for the hearing aid research community. The database is available under http://medi.uni-oldenburg.de/hrir/.

\section{Acknowledgment}

The authors would like to thank Siemens Audiologische Technik for providing the hearing aids and the appropriate equipment. This work was supported by the DFG (SFB/TR 31) and the European Commission under the integrated project DIRAC (Detection and Identification of Rare Audiovisual Cues, IST-027787).

\section{References}

[1] R. O. Duda and W. L. Martens, "Range dependence of the response of a spherical head model," The Journal of the Acoustical Society of America, vol. 104, no. 5, pp. 3048-3058, 1998.

[2] G. F. Kuhn, "Model for the interaural time differences in the azimuthal plane," The Journal of the Acoustical Society of America, vol. 62, no. 1, pp. 157-167, 1977.

[3] V. R. Algazi, R. O. Duda, D. M. Thompson, and C. Avendano, "The CIPIC HRTF database," in IEEE ASSP Workshop on Applications of Signal Processing to Audio and Acoustics, pp. 99102, October 2001.

[4] B. Gardner, K. Martin, et al., "HRTF measurements of a KEMAR dummy-head microphone," Tech. Rep. 280, MIT Media Lab Perceptual Computing, May 1994.

[5] S. Takane, D. Arai, T. Miyajima, K. Watanabe, Y. Suzuki, and T. Sone, "A database of head-related transfer functions in whole directions on upper hemisphere," Acoustical Science and Technology, vol. 23, no. 3, pp. 160-162, 2002.

[6] H. Sutou, "Shimada laboratory HRTF database," Tech. Rep., Shimada Labratory, Nagaoka University of Technology, Nagaoka, Japan, May 2002, http://audio.nagaokaut.ac.jp/hrtf.

[7] H. Puder, "Adaptive signal processing for interference cancellation in hearing aids," Signal Processing, vol. 86, no. 6, pp. 1239-1253, 2006.

[8] “Head and Torso Simulator(HATS) - Type 4128," Brüel \& Kjær, Nærum, Denmark.

[9] D. Berg, SoundMex2, HörTech gGmbH, Oldenburg, Germany, 2001.

[10] S. D. Ewert and H. Kayser, "Modified inverse repeated sequence," in preparation.

[11] D. D. Rife and J. Vanderkooy, "Transferfunction measurement with maximum-lengthsequences," Journal of Audio Engineering Society, vol. 37, no. 6, pp. 419-444, 1989.

[12] A. Farina, "Simultaneous measurement of impulse response and distortion with a swept-sine technique," in AES 108th Convention, Paris, France, February 2000.

[13] C. Dunn and M. Hawksford, "Distorsion immunity of mlsderived impulse response measurements," Journal of Audio Engineering Society, vol. 41, no. 5, pp. 314-335, 1993.

[14] J. Blauert, Räumliches Hören, Hirzel Verlag, 1974.

[15] L. Rayleigh and A. Lodge, "On the acoustic shadow of a sphere," Proceedings of the Royal Society of London, vol. 73, pp. 65-66, 1904.

[16] W. M. Rabinowitz, J. Maxwell, Y. Shao, and M. Wei, "Sound localization cues for a magnified head: implications from sound diffraction about a rigid sphere," Presence, vol. 2, no. 2, pp. 125-129, 1993.

[17] J. Nix and V. Hohmann, "Sound source localization in real sound fields based on empirical statistics of interaural parameters," The Journal of the Acoustical Society of America, vol. 119, no. 1, pp. 463-479, 2006.

[18] R. S. Woodworth and H. Schlosberg, Woodworth and Schlosberg's Experimental Psychology, Holt, Rinehardt and Winston, New York, NY, USA, 1971.

[19] M. R. Schroeder, "New method of measuring reverberation time," The Journal of the Acoustical Society of America, vol. 36, no. 3, pp. 409-413, 1964.

[20] M. Karjalainen and P. Antsalo, "Estimation of modal decay parameters from noisy response measurements," in AES 110th Convention, Amsterdam, The Netherlands, May 2001.

[21] T. Rohdenburg, S. Goetze, V. Hohmann, K.-D. Kammeyer, and B. Kollmeier, "Objective perceptual quality assessment for self-steering binaural hearing aid microphone arrays," in Proceedings of IEEE International Conference on Acoustics, Speech, and Signal Processing (ICASSP '08), pp. 2449-2452, Las Vegas, Nev, USA, March-April 2008. 